Cahiers $d u$ MONDE RUSSE

\section{Cahiers du monde russe}

Russie - Empire russe - Union soviétique et États indépendants

$46 / 4 \mid 2005$

L'invention d'une politique humanitaire

\title{
L'organisation non gouvernementale, Nouvel acteur du champ humanitaire
}

Le Zemgor et la Société des Nations dans les années 1920

\section{Dzovinar KÉVONIAN}

\section{(2) OpenEdition}

Journals

Édition électronique

URL : https://journals.openedition.org/monderusse/9429

DOI : $10.4000 /$ monderusse. 9429

ISSN : $1777-5388$

Éditeur

Éditions de l'EHESS

Édition imprimée

Date de publication : 1 décembre 2005

Pagination : 739-756

ISBN : 2-7132-2057-2

ISSN : $1252-6576$

Référence électronique

Dzovinar KÉVONIAN, "L'organisation non gouvernementale, Nouvel acteur du champ humanitaire », Cahiers du monde russe [En ligne], 46/4 | 2005, mis en ligne le 01 janvier 2005, consulté le 03 septembre 2022. URL : http://journals.openedition.org/monderusse/9429; DOI : https://doi.org/ $10.4000 /$ monderusse. 9429 


\title{
L'ORGANISATION NON GOUVERNEMENTALE, NOUVEL ACTEUR DU CHAMP HUMANITAIRE
}

\author{
Le Zemgor et la Société des Nations \\ dans les années 1920
}

Cette étude est une contribution à l'écriture d'une histoire des organisations communautaires créées par les réfugiés dans l'Europe de l'entre-deux-guerres et du rôle de ces organisations dans l'émergence d'une politique humanitaire. Nous examinerons ici le cas du Zemgor et plus précisément un aspect spécifique de son action dans les années 1920, à savoir sa place et ses orientations dans le cadre de l'action internationale menée en faveur des réfugiés russes par la Société des Nations (ci-après SDN), notamment au sein du Haut commissariat pour les réfugiés dirigé par Fridtjof Nansen à partir de 1921. Quelles furent les options prises individuellement et collectivement par le Zemgor dans le domaine humanitaire ? En quoi étaient-elles novatrices ou relevaient-elles d'une mutation en cours, sachant que la notion de modernité peut être déclinée soit en termes d'innovation soit en termes d'adaptabilité dans les pratiques, les concepts en action ou les représentations. Notre problématique sera la suivante : à travers l'exemple du Zemgor et de ses rapports avec les instances de la SDN chargées de la question des réfugiés, analyser les relations entre une organisation non gouvernementale et une organisation internationale dans cette phase particulière des années vingt qui voit s'institutionnaliser l'action humanitaire internationale.

Dans cet article, la graphie retenue pour les noms russes est celle des documents d'archives consultés. 


\section{Structuration de l'action humanitaire internationale face aux nécessités}

Le début des années vingt voit se conjuguer la mutation de la question des réfugiés et l'apparition de structures internationales spécialisées ${ }^{1}$. Des mouvements de masse de populations victimes des idéologies ou des recompositions territoriales issues de la Première Guerre mondiale se substituent au nombre limité des exilés politiques du XIX ${ }^{\mathrm{e}}$ siècle. Avec le XXe siècle, la généralisation de l'apatridie, la pratique des dénaturalisations pour raisons politiques et l'apparition des échanges forcés de populations transforment la question des réfugiés en un «véritable problème de politique internationale ». Par ailleurs, l'action internationale menée en faveur des réfugiés est alors intimement liée à la perception et l'approche que les différents acteurs ont des nombreux problèmes posés par ces réfugiés. Le réfugié apatride apparaît défini avant tout par son « infirmité » légale, l'absence de nationalité. Il renvoie une image négative ${ }^{2}$. La structure des relations internationales et les mentalités collectives conditionnent également l'appréhension de la question des réfugiés. L'organisation de la société internationale, le dogme de la souveraineté et de l'égalité des États contribuent à faire du réfugié apatride un danger social dont la prise en charge par la Société des Nations devient une nécessité. Cette perception du problème détermine les solutions : le rapatriement apparaît comme une priorité. À défaut, on envisage le placement collectif ou individuel dans un pays d'accueil. De la même manière, le problème est perçu comme temporaire, devant être réglé au cas par cas. De là découle la classification en catégories nationales adoptée par la SDN à partir de 1922, d'abord au profit des réfugiés russes ${ }^{3}$. Enfin, il s'agit d'une question technique et non politique. Cette distinction est rigoureusement défendue au sein du Conseil de la SDN au nom du respect du statu quo politique européen et du respect de l'indépendance étatique. Cependant, au lendemain de la Première Guerre mondiale, un certain nombre de juristes internationalistes en marge de ces perceptions dominantes se livrent à une large critique des nouvelles conditions faites aux hommes dans ce monde où, disent-ils, le droit des gens n'a jamais été tant bafoué. Selon eux, les réfugiés apatrides sont les manifestations les plus criantes de ces évolutions qu'ils considèrent comme une régression des droits naturels de l'homme. Leurs travaux les conduisent à élaborer les droits internationaux de l'homme en partant de réflexions simultanées et concordantes sur la question du droit des réfugiés, des minorités et du droit international humanitaire ou droit de Genève. En 1930, la Conférence de La Haye relative à la codification du droit inter-

\footnotetext{
1. Dzovinar Kévonian, Réfugiés et diplomatie humanitaire : les acteurs européens et la scène proche-orientale pendant l'entre-deux-guerres, Paris : Publications de la Sorbonne, 2004.

2. Marc Vichniac, « Le statut international des apatrides », Recueil des Cours de l'Académie de droit international de La Haye, $n^{\circ} 43,1933$, p. 119-245.

3. Louise Holborn, « The Legal Status of Political Refugees (1920-1938) », American Journal of International Law, 32, 1938, p. 680-703 ; James Hathaway, « The Evolution of Refugee Status in International Law (1920-1950) », International and Comparative Law Quarterly, 33, 1984, p. 348-380
} 
national qui définit les droits internationaux de l'homme marque le point culminant de ce mouvement ${ }^{4}$.

Parallèllement, l'action humanitaire internationale subit de la même manière les bouleversements induits par la guerre. Au-delà de l'immensité de la tâche et des souffrances humaines générées par ce conflit, la structure du champ humanitaire est modifiée par les nouveaux rapports de force de l'après-guerre. L'évolution de l'action humanitaire fait coexister différentes « strates » : passage de la charité à la philanthropie, émergence et codification d'un droit international humanitaire par le Comité international de la Croix-Rouge, élaboration de la théorie de l'intervention d'humanité en connexion avec les différentes phases de la Question d'Orient, montée en puissance de la philanthropie américaine, essor de l'humanitaire d'État en liaison avec le développement de la médecine coloniale et militaire, etc., avec, à partir de 1921, l'apparition au sein de la SDN d'une structure spécialisée chargée de la question des réfugiés. Nouvel organe central, le Haut commissariat se veut désormais le pôle fédérateur de l'ensemble des initiatives publiques et privées. Il s'agit là de la naissance d'une diplomatie humanitaire en temps de paix, caractérisée par l'élargissement progressif de ses compétences selon une classification des réfugiés par nationalité et une approche empirique des problèmes posés. Néanmoins, l'absence de moyens propres et les limites de la coopération internationale brident la structure en dépit de la personnalité imposante de Fridtjof Nansen. À partir de 1925, le BIT prend en charge le placement professionnel des réfugiés. Cette approche technique et économique place la question des réfugiés hors du champ politique. Considérée comme un problème social, cette question est traitée avec pragmatisme par une organisation internationale à caractère technique. Dans le même temps, Fridtjof Nansen consacre une grande partie de son énergie à mettre en place la reconnaissance d'un véritable statut juridique aux réfugiés et apatrides et à étendre l'usage du certificat d'identité créé en 1922 pour les réfugiés russes à d'autres catégories de réfugiés 5 .

Dans ces années-là, le dernier aspect de l'action du Haut commissariat pour les réfugiés se caractérise par l'essor d'une pratique diplomatique et la production d'un droit. Le rôle original du Comité consultatif des organisations privées mis en place par Fridtjof Nansen et les débuts d'une pratique qui associe les acteurs non gouvernementaux à la coopération internationale relève d'une expérience novatrice qui mérite d'être soulignée. En effet, après 1945, les évolutions propres à la période ont vu de telles pratiques se renouveler et aussi faire l'objet d'une reconnaissance et d'une normalisation juridique. Sur le plan du droit, le haut commissaire a posé les jalons du statut international des réfugiés avec, pour élément décisif ayant valeur de point d'orgue, la conclusion de la convention de 1933. Ainsi, et bien que les questions sociales ne comptent pas parmi ses priorités, la SDN nouvellement créée

4. J. Guerrero, « La conférence de La Haye relative à la codification du droit international (13 mars-12 avril 1930) », Revue de Droit international, $n^{\circ} 5,1930$, p. 478-491, n 6, 1930, p. 649667

5. Claudena M. Skran, Refugees in Inter-War Europe: The Emergence of a Regime, Oxford : Clarendon Press, 1995. 
devient néanmoins le lieu où s'élaborent des outils juridiques, où se développent des pratiques coopératives et où les organisations privées et les milieux d'experts trouvent une tribune d'expression et un lieu d'action nouveaux dans un champ traditionnellement réservé aux acteurs étatiques. La SDN, sujet dérivé de la société internationale et première organisation internationale à vocation universelle, accélère ainsi son processus d'autonomie juridique, de légitimation et de conquête de son propre champ. Au regard de ces évolutions et de la recomposition du champ humanitaire au début des années vingt, nous déterminerons les formes spécifiques des relations entre la SDN et le Zemgor.

\section{Lieux et domaines de collaboration : de la vigilance normative à l'action opérationnelle}

On sait l'expérience acquise par les structures fédérées au sein du Zemgor pendant la guerre : action sociale et de secours, aide aux réfugiés et aux personnes déplacées, coordination et gestion d'une « crise humanitaire ». La première étape de la collaboration entre la SDN et le Zemgor date de 1920, lorsque l'on confie à l'explorateur et philanthrope norvégien Fridtjof Nansen la gigantesque opération de rapatriement des prisonniers de guerre. L'opération consiste à organiser le rapatriement des prisonniers de guerre et des internés civils des empires centraux toujours en Sibérie (350 000 personnes) et celui des prisonniers russes encore stationnés en Europe centrale. La situation de dénuement et de précarité des anciens prisonniers russes, en Allemagne en particulier, est alarmante. Le Comité international de la Croix-Rouge (ci-après CICR) évalue leur nombre à 200000 personnes, réparties en mars 1920 dans 43 camps d'internement ${ }^{6}$. Les opérations sont menées en coopération avec le CICR et le YMCA. Elles durent de mai 1920 à juillet 1922 et permettent à 430000 personnes de réintégrer leurs foyers.

Au début de l'année 1921, avec la victoire bolchevique, c'est la question des réfugiés russes qui devient une urgence internationale. La situation est particulièrement dramatique à Constantinople. De Paris, le prince Lvov, président du Comité directeur du Zemgor, écrit en mai 1921 au président du Conseil de la SDN pour demander une réaction institutionnelle forte. À Genève, le CICR insiste auprès du Conseil de la SDN pour une prise en charge de la question des réfugiés russes et obtient la nomination de Fridtjof Nansen comme haut commissaire de la SDN pour les réfugiés russes en juin 1921. Sa mission consiste à favoriser une meilleure coordination des initiatives privées et à organiser une conférence intergouvernementale chargée de trouver des solutions au problème de l'absence de statut juridique clair de ces réfugiés. Le 23 août 1921, s'ouvre à Genève la première conférence d'étude sur la question des réfugiés russes. Dès cette première conférence, le Zemgor de Paris, nouvellement constitué, devient avec la Croix-Rouge russe représentée par le

6. Le Comité international de la Croix-Rouge : ses missions (1918-1923), Genève : CICR, 1923. 
docteur Georges Lodygenski ${ }^{7}$, le partenaire principal du haut commissaire. Dès le départ également, les juristes russes comme André Mandelstam (membre du Conseil des anciens ambassadeurs de Russie) et Constantin Goulkevitch sont partie prenante du processus d'élaboration d'un statut juridique pour les réfugiés. L'absence d'une législation unifiée demeure le principal problème des réfugiés auquel il faut adjoindre celui d'une prise en charge inégale dans les différents pays d'accueil, selon qu'ils sont considérés comme un problème national ou international. Une collaboration active s'établit et se structure autour de l'action juridique et du placement professionnel. La question de la catégorisation des apatrides est au centre des préoccupations qui agitent le HCR comme le Zemgor.

En créant le Comité consultatif des organisations privées près le Haut commissariat (ci-après CCOP) pour les réfugiés en octobre1921, Fridtjof Nansen fonde un lieu d'échange pionnier de relations entre l'organisation internationale, des organisations privées humanitaires et des organismes communautaires de réfugiés. Le Zemgor est alors représenté par son délégué à Genève, Nicolas I. Astrov, qui est membre du Comité directeur de l'Union des villes. Il est bien connu pour avoir été à l'initiative du Centre des archives russes à l'étranger de Prague. Les bases de la collaboration instituée entre le CCOP et le HCR sont en grande partie fixées par Lucien Wolf, célèbre représentant de l'émigration juive, délégué de la Jewish Colonisation Association et du Joint Foreign Committee de Londres $^{8}$. Les organisations privées communautaires et généralistes garderont leur autonomie absolue dans leur champ d'action ainsi que le contrôle total de leur fonds propres. En ce qui concerne les ONG, l'objectif du CCOP est donc de centraliser les informations auprès du Haut commissariat afin de coordonner les activités et d'empêcher le «gaspillage des efforts ». Par ailleurs, il est admis que certaines questions d'intérêt général, comme la gestion des mouvements migratoires ou l'élaboration d'un statut juridique international pour les réfugiés, ne peuvent être résolues que par le HCR. Le haut commissaire peut également stimuler les appels de fonds et se faire le relais officiel des besoins financiers des organisations privées. Enfin, le CCOP doit être un lieu d'échange des informations en particulier sur les urgences humanitaires. C'est d'ailleurs le cas en novembre 1921 pour la situation à Constantinople, comme l'atteste la correspondance entre F. Nansen et N. Astrov. Le Comité des zemstvos et villes russes à Genève alerte Nansen sur la situation dramatique des réfugiés russes à Constantinople depuis le départ de la Croix-Rouge américaine et soulève notamment le problème de la prostitution des femmes ${ }^{9}$. Nansen fait intervenir son délégué local Georges Burnier et obtient des Haut commissaires alliés la délivrance de visas permettant aux femmes concernées de quitter la capitale ottomane ${ }^{10}$. Il lance égale-

7. Il est également le secrétaire général du Comité national russe de secours aux enfants, affilié à l'Union internationale de secours aux enfants.

8. Conférence des réfugiés russes. Réunion entre le Haut commissaire et les représentants des associations privées, 19 septembre 1921. Archives de la Société des Nations (ci-après ASDN), Fonds Mixte Nansen (ci-après FMN), CRR/PV extraordinary.

9. N. Astrov à F. Nansen, 4 novembre 1921. ASDN, FMN, CCRR/OP/12.

10. F. Nansen aux Hauts Commissaires alliés, 9 novembre 1921. ASDN, FMN, CCRR/OP/12. 
ment un appel de fonds auprès d'une dizaine d'organisations internationales privées agissant pour la protection des femmes. La gravité de la situation à Constantinople aboutit également à la rédaction d'une note collective de toutes les associations membres du CCOP adressée au haut commissaire Nansen et au délégué général de la SDN réclamant une « action immédiate de secours $»^{11}$.

Cette collaboration se structure à partir de 1922 et prend toute son ampleur entre 1923 et 1930. C'est l'âge d'or du Comité consultatif des organisations privées dont le déclin s'amorce après la mort de Nansen en 1930 et la restructuration du Haut commissariat pour les réfugiés. Les relations sont étroites et très actives entre le haut commissaire et le Zemgor (représenté en tant que tel au CCOP et au travers d'une dizaine d'associations proches ou directement associées à lui). On retrouve ainsi à Genève tout au long des années vingt Nicolas Astrov, Vadim Rudnev (ancien membre du Comité central du parti S.-R., secrétaire de la Tribune juive, fondateur de Sovremennye zapiski), Nicolas Avksentieff également issu du parti S.-R. et, prenant activement part aux débats genevois, Jacob Rubinstein, avocat social-démocrate, un temps menchevik internationaliste et ancien président de la Douma de Kharkhov. Ce dernier demeure à Berlin et devient membre du Comité directeur du Zemgor à partir de 1922. Le Zemgor de Prague et celui de Belgrade sont également représentés à Genève par Vladimir Lebedeff, Ivan Brouchvit et Théodore Makhine. La proximité politique des responsables du Zemgor de Paris (pour la plupart libéraux issus du parti K.-D.) avec les ambassadeurs et diplomates mandatés par le dernier gouvernement russe, réunis au sein du Conseil des ambassadeurs, se traduit par l'importance de leur représentation et de leur influence dans les relations entretenues avec Nansen et les instances spécialisées de la SDN. Constantin Goulkevitch incarne ce lien pendant plus de dix années. Diplomate de formation, il a été en poste à Stockholm puis à Constantinople jusqu'en 1914. Installé dans le Valais suisse dans les années vingt, il est au centre d'un réseau quadrangulaire entre Paris, Prague, Berlin et Genève. Enfin, le rôle déterminant que jouent un certain nombre de juristes russes ou juifs russes comme experts associés lors de l'élaboration du certificat d'identité et de voyage de 1922 (plus connu sous le nom de «passeport Nansen ») puis lors des conférences intergouvernementales de 1926, 1928 et 1933, caractérise au mieux la relation étroite qui unit le Zemgor et le Haut commissariat. Ainsi, dès 1921, la Commission centrale pour l'étude de la question des réfugiés est créée à Paris au sein de la Conférence des ambassadeurs. Cette commission, composée de juristes russes et juifs russes dont André Mandelstam, le baron Boris Nolde, Constantin Goulkevitch, Jacob Rubinstein, Boris Mirkine-Guetzevitch, Alexandre Konovalov, Vadim Roudneff et V. F. Zeeler, traduit la convergence d'intérêt qui prévaut alors dans les milieux libéraux sur la mise en place d'un statut générique commun pour les réfugiés de l'ancien empire russe ${ }^{12}$. Son importance dépasse d'ailleurs largement les liens 
entre les organisations communautaires russes et le Haut commissariat pour les réfugiés. Dès février 1920, ses membres avaient fondé l'Association russe pour la SDN à Paris $^{13}$. On les retrouve membres de la Ligue russe des droits de l'homme affiliée à partir de 1922 à la Fédération internationale des droits de 1'homme ${ }^{14}$ et présents au sein de l'Institut de droit international et de l'Académie de droit de La Haye. Ils participent aux grandes évolutions théoriques qui marquent le droit international pendant l'entre-deux-guerres ${ }^{15}$, contribuant ainsi à la maturation de concepts juridiques qui dépassent par leur portée historique les résultats concrets et limités de leur collaboration avec le $\mathrm{HCR}^{16}$.

\section{Redéploiement des populations et migrations dirigées}

Lorsque de 1925 à 1929, après l'échec avéré des opérations de rapatriement ${ }^{17}$, l'action internationale en faveur des réfugiés est transmise au Bureau international du travail (ci-après BIT) afin de résoudre les problèmes liés au chômage des réfugiés, le Zemgor va montrer sa capacité d'adaptation. Il poursuit et approfondit les échanges avec le HCR afin d'améliorer le statut juridique des réfugiés apatrides, mais sait également saisir au bond l'occasion que représente le placement professionnel coordonné par Albert Thomas, l'actif directeur du BIT. Dans la correspondance régulière qu'il a établie avec Fridtjof Nansen, Nicolas Astrov présente avec clarté la nouvelle situation des réfugiés russes et met lui-même en avant l'intérêt d'une collaboration avec le BIT. Il explique ainsi que la phase d'urgence et de charité est révolue et que le problème de l'émigration russe doit désormais être conçu sur le long terme :

13. Présidents : N. Avksentieff, M. Konovalov, M. Vinaver ; secrétaires généraux : A. Mandelstam, V. Roudneff, J. Rubinstein ; membres du comité central: prince Lvov, A. Michelson, Baron B. Nolde, M. Vichniac, M.Zenzinov. Statuts de l'association dans Tribune juive, $\mathrm{n}^{\circ} 10,5$ mars 1920 .

14. BDIC, Archives de la Ligue des droits de l'homme, F delta, rés. 798/60. Dirigée par N. Avksentieff, B. Mirkine-Guetzevitch en est le premier secrétaire général. Sont également membres de la Ligue russe : P. Milioukov, J. Rubinstein et O. Minor.

15. Voir en particulier les travaux d'André Mandelstam en droit international et ceux de Boris Mirkine-Guetzevitch en droit constitutionnel.

16. D. Kévonian, «Exilés politiques et avènement du "droit humain" : la pensée juridique d'André Mandelstam (1869-1949) », Revue d'Histoire de la Shoah, n 177-178, janvier-août 2003, p. 245-273.

17. F. Nansen abandonne en 1924 les tentatives de rapatriement des réfugiés russes en URSS. Voir son rapport aux membres du Conseil de la SDN en date du 24 juin 1924. Voir également le mémorandum officiel du Zemgor sur la question du rapatriement, signé de Nicolas Astrov et transmis à la SDN en septembre 1923. ASDN, FMN, R1738, 17822: « Le problème des réfugiés n'aurait perdu de son acuité et n'aurait cessé de se présenter comme un problème international que dans deux cas : 1/ si les conditions de la vie en Russie avaient suffisamment changé pour donner la possibilité à la majorité des réfugiés de revenir à leurs foyers et 2/ si les réfugiés, dispersés dans de nombreuses contrées, étaient tant soit peu assimilés à la population indigène, en étaient devenus, pour ainsi dire, une partie organique. Ni l'un, ni l'autre de ces faits ne peut être considéré comme aquis ». 
Les réfugiés sont devenus après quatre ans de séjour en Europe des émigrés, obligés à organiser de leur mieux leur existence en pays étranger pour une période dont la durée ne saurait être déterminée ${ }^{18}$.

L'analyse de l'action générale de placement entre 1925 et 1929 permet de dresser un bilan des relations avec le Zemgor et de mettre en lumière les éléments suivants. Ainsi, pour la première fois depuis le début des années vingt, le BIT, organisation internationale, est chargé d'une fonction exécutive : il ne doit plus seulement coordonner les activités des organisations privées et servir d'interface avec les représentants étatiques ${ }^{19}$, il doit aussi placer le plus grand nombre de réfugiés. Or, le BIT ne dispose que de peu de moyens opérationnels, contrairement au Zemgor qui présente cet aspect comme son atout majeur. Les organisations affiliées au Zemgor sont en effet présentes dans les pays d'accueil des réfugiés. Elles ont déjà une pratique des bureaux de placements (à Prague et à Paris notamment) et disposent de filières d'immigration par leurs contacts avec des sociétés privées. Nicolas Astrov parle ainsi de l'existence de bureaux de travail en France (Paris, Marseille), en Allemagne, Pologne, Estonie, Finlande, Turquie, Bulgarie, Grèce et Tunisie et du placement de plus de 40000 personnes en quatre ans. Mais selon lui, la difficulté repose sur le fait que la moitié des personnes placées exerçaient précédemment des professions intellectuelles et qu'elles ont dû accepter des travaux de nature physique ou manuelle qui ne leur correspondent pas. C'est notamment le cas dans les exploitations forestières en Estonie, Pologne et Finlande. De même en Bulgarie ou en Yougoslavie :

Dans ces pays, les intellectuels russes se sont déjà habitués au travail physique et ne le fuient pas. Mais il est tout naturel qu'un étudiant, un avocat ou un procureur, qui pousse la brouette dans les mines de houille de Pernic en Bulgarie, ou traîne des briques sur son dos pour des bâtiments élevés à Sofia, rêve au bonheur indicible de travailler dans une usine quelconque de la France ou de la Belgique ${ }^{20}$.

De manière plus générale, Nicolas Astrov répartit en 1924 les pays d'accueil en deux groupes : ceux où la situation économique et sociale des réfugiés est convenable (France, Belgique, Suisse, Angleterre, Italie, Tchécoslovaquie, Lituanie) et ceux où les problèmes restent importants (Allemagne, Pologne, Finlande, Estonie, Lettonie, Yougoslavie, Bulgarie, Grèce, Turquie, Chine). La solution réside donc dans un redéploiement des populations réfugiées : «Une distribution bien organisée d'une partie des ouvriers russes des pays précités parmi les pays ayant une plus grande demande de travail, faciliterait extrêmement la situation ». En conséquence, Nicolas Astrov

18. N. Astrov à F. Nansen, 5 août 1924. ASDN, FMN, R1738, 17822.

19. Le mandat accepté par le BIT et qui prend effet à partir de janvier 1925, comprend la recherche, la coordination et la communication des offres de placements qui peuvent être offertes aux réfugiés russes et arméniens, des conditions dans lesquelles les réfugiés peuvent profiter de ces offres et une évaluation statistique par pays des réfugiés à placer. Procès-verbal de la $5^{\mathrm{e}}$ Commission de la Ve Assemblée générale de la SDN, 12 septembre 1924. Archives du ministère français des Affaires étrangères (ci-après AMAE), SDN, 1804, fo 1-2. 
considère que le BIT pourrait faciliter ces transferts de main-d'œuvre qui seraient organisés par le Zemgor. Le BIT pourrait également se charger de trouver l'argent nécessaire à l'évacuation des réfugiés russes de Kharbin (Chine) et négocier avec les États-Unis des possibilités d'émigration. Pour Albert Thomas, un tel montage n'est pas envisageable. Il ne s'agit pas pour le BIT de mettre en place une « collaboration étroite » avec le Zemgor. Le BIT en tant que secrétariat permanent de l'Organisation internationale du travail doit être l'unique maître d'œuvre des opérations de placement et, dans la lettre officielle qu'il adresse au prince Lvov, A. Thomas remplace la notion de «collaboration étroite » par cette formule : «votre expérience peut nous être précieuse $»^{21}$. Quelques jours plus tard, Albert Thomas reçoit également une longue note du Zemgor de Prague, signée d'Ivan Brouchvit, présentant l'expérience de la section du travail du Zemgor en Tchécoslovaquie en matière de placement professionnel ${ }^{22}$. En dépit de l'insistance du Zemgor et de sa réelle expérience opérationnelle, Albert Thomas entend faire diriger les opérations de placement par le BIT et met en place un système centralisé d'information et de négociation à Genève (Bureau des réfugiés) relayé sur le terrain par les délégués du BIT qui constitueront des comités consultatifs locaux des organisations russes, dont le Zemgor. En 1925, deux logiques concurrentes se font face : celle de l'organisation internationale et celle de l'organisation non gouvernementale. Albert Thomas ne considère pas l'OIT et notamment son secrétariat permanent comme des institutions de simple expertise scientifique et d'enquêtes sur les conditions de travail. Son action est sous-tendue par des convictions profondes : la nécessité de remplacer une économie du profit par une économie du travail, gage d'une véritable justice sociale, elle-même seule susceptible de garantir une paix universelle. Son interprétation du mandat confié à l'OIT depuis 1919 l'amène ainsi à considérer l'organisation internationale certes comme une institution normative, mais également comme un centre opérationnel de politiques publiques internationales. Ainsi, la question des migrations et du placement des réfugiés sans emploi s'intègre dans un raisonnement plus large sur les équilibres macrosociaux régionaux (Europe) et intercontinentaux en rapport avec les questions du chômage et de l'adoption des législations sociales ${ }^{23}$. Fort de son expérience d'économie dirigée en 1916-1917 au ministère des Armements et des Équipements de guerre, A. Thomas se pose en maître d'œuvre d'une opération de placement international des réfugiés russes conçue comme une première expérimentation d'immigration « internationale » dirigée. Le système qu'il entend mettre en place est fondé sur des accords conclus avec quatre types d'interlocuteurs : les délégués nationaux du BIT (identification et quantification de l'offre dans les pays-sources et de la demande dans les pays d'accueil) ; les représentants étatiques qualifiés (ministères du Travail et/ou de l'Agriculture, services de la main-d'œuvre étrangère); les représentants

21. A. Thomas au prince Lvov, 15 octobre 1924. ASDN, FMN, C1437, R 404/3/22/1.

22. I. Brouchvit à A. Thomas, 22 octobre 1924. ASDN, FMN, C1436, R 404/1/1/1.

23. Le BIT accomplit un « travail de caractère purement technique, qui fait de lui une chambre de compensation internationale pour les offres et les demandes d'emploi des réfugiés dans les divers pays », déclare Albert Thomas. "L'œuvre de secours aux réfugiés », Revue Internationale du Travail, 12(1), janv. 1928, p. 83. 
patronaux ou organisations patronales sectorielles (identification des besoins et des conditions d'embauche); les compagnies d'immigrations privées (notamment la célèbre Société générale d'immigration). Son atout réside dans les contacts déjà établis pendant la guerre et les réseaux dont il dispose en France. Dans cette perspective, une collaboration avec le Zemgor ne peut donc être que locale (pays-sources) par son intégration dans des comités consultatifs près des différents délégués du BIT ${ }^{24}$.

\begin{tabular}{|c|c|}
\hline $\begin{array}{l}\text { Délégués successifs } \\
\text { du BIT (1925-1928) }\end{array}$ & Pays concernés \\
\hline A. Czamanski & France (Paris). Bénévole. \\
\hline $\begin{array}{l}\text { J. Charpentier puis } \\
\text { I. Arnould }\end{array}$ & $\begin{array}{l}\text { Pologne (Varsovie), assisté d'une commission consultative et de } \\
\text { sélection composée de représentants des organisations de réfu- } \\
\text { giés russes. }\end{array}$ \\
\hline H. Cuénod & $\begin{array}{l}\text { Chine (Kharbin puis Shangaï), assisté par les organisations euro- } \\
\text { péennes de secours. }\end{array}$ \\
\hline E. Gallati & $\begin{array}{l}\text { Estonie, Lettonie, Lituanie (Riga), assisté d'une commission } \\
\text { consultative et de sélection composée de représentants des orga- } \\
\text { nisations de réfugiés. }\end{array}$ \\
\hline A. Mazaryk & $\begin{array}{l}\text { Tchécoslovaquie (présidente de la Croix-Rouge tchécoslovaque, } \\
\text { Prague), assistée d'une commission consultative et de sélection } \\
\text { composée de représentants des organisations de réfugiés. Bénévole. }\end{array}$ \\
\hline $\begin{array}{l}\text { H. Reymond puis } \\
\text { Dr M. Heimroth }\end{array}$ & $\begin{array}{l}\text { Autriche, Hongrie (Vienne), assisté d'une commission consulta- } \\
\text { tive composée de représentants des autorités locales et des orga- } \\
\text { nisations de réfugiés. }\end{array}$ \\
\hline S. L. Childs & Argentine (Buenos-Aires) \\
\hline $\begin{array}{l}\text { A. Scherbatskoy puis } \\
\text { M. Schlesinger }\end{array}$ & $\begin{array}{l}\text { Allemagne (Berlin), assisté par les organisations locales de réfu- } \\
\text { giés russes. }\end{array}$ \\
\hline Maj. Gén. Schwindt & $\begin{array}{l}\text { Finlande (Chef du cabinet militaire du président de la République } \\
\text { finlandaise ; président de la Croix-Rouge finlandaise, Helsing- } \\
\text { fors). Bénévole. }\end{array}$ \\
\hline \multicolumn{2}{|c|}{$\begin{array}{c}\text { Délégué général pour le Proche-Orient } \\
\text { Capt. S. L. Childs (Belgrade) puis R. Schlemmer (Istanbul) } \\
\text { Bulgarie, Yougoslavie, Roumanie, Grèce, Turquie, Syrie-Liban }\end{array}$} \\
\hline M. G. Zwerner & $\begin{array}{l}\text { Grèce (Salonique), assisté d'une commission consultative et de } \\
\text { sélection pour les réfugiés composée de représentants des auto- } \\
\text { rités locales et des organisations de réfugiés. }\end{array}$ \\
\hline M. Kotelnikoff & Grèce (Athènes) \\
\hline $\begin{array}{l}\text { M. Hesse puis } \\
\text { M. Lemtiougov }\end{array}$ & $\begin{array}{l}\text { Turquie (Istanbul), assisté d'une commission consultative et de } \\
\text { sélection composée de représentants des autorités locales et des } \\
\text { organisations de réfugiés. }\end{array}$ \\
\hline G. Burnier & $\begin{array}{l}\text { Syrie-Liban (Beyrouth); assisté du Comité central de secours aux } \\
\text { réfugiés arméniens (Beyrouth) et du Sous-Comité arménien } \\
\text { (Genève). }\end{array}$ \\
\hline M. Yourieff & Royaume des Serbes, Croates, Slovènes (Belgrade) \\
\hline M. Serafimoff & $\begin{array}{l}\text { Bulgarie (Sofia), assisté d'une commission consultative et de } \\
\text { sélection composée de représentants des autorités locales, des } \\
\text { organisations de secours et des organisations de réfugiés. }\end{array}$ \\
\hline
\end{tabular}

24. SDN et BIT, Rapport sur l'œuvre en faveur des réfugiés, sept. 1925. AMAE/P, SDN, 1802, $\mathrm{f}^{\circ} 22$. 
En mars 1925, utilisant la courroie de transmission du CCOP, il présente aux organisations privées les grandes lignes de l'opération telle qu'il la conçoit : « Je vous demande de dire à vos comités locaux de se mettre en rapport étroit avec les délégués, afin d'élaborer un programme unitaire ${ }^{25}$. À cette date, Albert Thomas a déjà conclu un accord déterminant pour la réussite du projet. Il a en effet obtenu du ministère français du Travail l'engagement d'avoir le monopole du recrutement des réfugiés russes pour des emplois industriels à destination de la France ${ }^{26}$. Il a par ailleurs un contact précieux au ministère français de l'Agriculture, en la personne de Marcel Paon qui est en charge des questions de main-d'œuvre agricole et qui partage ses idées en matière d'immigration dirigée ${ }^{27}$. Marcel Paon est membre du Conseil national de la main-d'œuvre et du Conseil supérieur de l'agriculture, expert près le Comité permanent de l'émigration du BIT. Pour Albert Thomas, ce système est avantageux pour tous les acteurs en présence (États de départ, États d'immigration, employeurs, réfugiés sans emploi) :

Ainsi, tout le recrutement se trouve centralisé et contrôlé de façon à empêcher tout monopole au bénéfice d'une organisation particulière de réfugiés d'un caractère politique ou l'exploitation de réfugiés par des intermédiaires ou autrement $^{28}$.

Souci de contourner les clivages politiques de l'émigration russe et volonté de soustraire les réfugiés à la toute-puissance des recruteurs de main-d'œuvre clandestine qui sévissent à Constantinople, Beyrouth ou Belgrade vont de pair.

Il est clair pour tous, et pour le Zemgor en particulier, que l'opération de placement international va se dérouler au profit de la France. Effectivement, de 1925 à 1929, du fait des relations et des réseaux d'Albert Thomas, la France se trouve au centre des opérations de placement, et ce malgré la crise de 1927 qui conduit les autorités françaises à réduire l'immigration de main-d'œuvre étrangère ${ }^{29}$. En dépit des divergences initiales, le Zemgor se rallie rapidement au programme mis en place par le BIT. Ce ralliement s'explique par le fait que depuis 1923, les autorités françaises ont mis fin à l'activité officielle des bureaux de placement du Zemgor sur

25. Réunion du Comité consultatif des organisations privées, 9 mars 1925. AMAE/P, SDN, $1804, \mathrm{f}^{\circ} 18-34$.

26. Le détail de l'accord entre M. Lebelle (service de la main-d'œuvre étrangère) et A. Thomas a été présenté dans une autre étude : Dzovinar Kévonian, «Enjeux de catégorisations et migrations internationales : le Bureau International du Travail et les réfugiés (1925-1929) », Revue européenne des migrations internationales, 21(3), 2005, p. 95-122.

27. Voir l'ouvrage de Marcel Paon sur la question, publié en 1926 et la préface d'Albert Thomas qui l'introduit : L'Immigration en France (Paris : Payot).

28. Mémoire présenté par le Directeur du BIT, Albert Thomas, 3 sept. 1926. ASDN, FMN, C1470, A.44.1926

29. Environ 20000 réfugiés russes sont placés en France dans cette période par le BIT. Résumé statistique du recensement, de l'évacuation et de l'établissement des réfugiés russes et arméniens du 1er janvier 1925 au 15 mai 1927. ASDN, FMN, C1400, R 402/10/0 ; BIT/SDN, rapport annuel de 1928. ASDN, FMN, C1471, A.33.1928.VIII. 
le territoire national (Paris, Marseille) ${ }^{30}$ et que l'organisation peut trouver un intérêt certain dans l'opération menée par le BIT. Par ailleurs en 1924, Le Comité directeur du Zemgor est partisan d'une immigration dirigée vers la France pour des raisons d'ordre politique :

Les Russes s'y sentent citoyens libres. Ils n'y sont soumis à aucun régime spécial d'émigrés et ils sont à l'abri des influences des organisations militaires et politiques aux tendances monarchiques et révolutionnaires ${ }^{31}$.

Signalons enfin que ce ralliement ne signifie pas l'intégration au programme genevois. Ainsi, dès le mois de février 1925, les représentants du Comité des zemstvos et villes russes au sein du CCOP annoncent la création d'une commission au sein du Zemgor chargée de gérer le problème du transfert en France des réfugiés russes résidant dans d'autres pays européens ${ }^{32}$. En novembre 1926, le Comité de Paris crée une commission spécifique pour le placement agricole en France des réfugiés russes. Quelques mois plus tôt, alors qu'Albert Thomas revenait d'un voyage d'étude en Amérique du Sud où il envisageait d'organiser le placement d'une partie des réfugiés russes, Nicolas Astrov s'est empressé de proposer la création par le Zemgor d'un comité de colonisation russe en Amérique du Sud. Une fois encore, le directeur du BIT a tenu à remettre les choses au point sur « sa » politique à l'égard des associations russes :

Il faut être extrêmement poli et gentil. Il faut garder leur concours en toute circonstances. Mais il ne faut pas les laisser empiéter dans l'œuvre dont nous avons la charge et la responsabilité.

Il est donc nécessaire, explique-t-il au responsable du Service des réfugiés du BIT d'éviter la formation d'un tel comité :

Réglez de la manière la plus large possible et la plus intelligente la collaboration des associations russes, mais n'abandonnons pas nos droits et nos responsabilités ${ }^{33}$.

L'insistance du Zemgor va alors se concentrer sur les attentes plus spécifiques de l'organisation au regard des difficultés des communautés russes d'Europe centrale et orientale. Ainsi, la question des étudiants russes s'affirme comme une préoccu-

30. C. Krovopouskoff (secrétaire général du Zemgor) à M. Lebelle (Service de la maind'œuvre étrangère du ministère français du Travail), 8 décembre 1923. ASDN, FMN, C1437, $\operatorname{Rr} 404 / 3 / 22 / 1$.

31. Prince Lvov à Albert Thomas, 30 septembre 1924. ASDN, FMN, C1437, Rr 404/3/22/1.

32. Sont membres de cette commission : les représentants des organisations cosaques (Conseil unifié du Don, du Kouban et du Terek, délégations en Tchécoslovaquie, Bulgarie et Yougoslavie), l'Union agricole (siège à Prague, délégations à Paris et en Yougoslavie). ASDN, FMN, C1437, $\operatorname{Rr} 404 / 3 / 22 / 1$.

33. Note d'Albert Thomas au major Johnson, 2 octobre 1925. ASDN, FMN, C1438, Rr 404/4/ $22 / 1$. 
pation majeure pour laquelle la collaboration du BIT est attendue. Le problème se pose en 1925 pour les nombreux étudiants qui se trouvent en Tchécoslovaquie et y terminent leur formation universitaire, formation qui avait été prise en charge par les autorités de Prague sous la forme du versement d'une indemnité34. Nicolas Astrov avait déjà saisi Fridtjof Nansen de cette question en 1923. Il évaluait alors le nombre total d'étudiants à 15000 dont la moitié étaient obligés d'occuper des emplois de manœuvres. En 1924, il présentait un état similaire de la question dans sa correspondance avec le BIT ${ }^{35}$. En décembre 1924, Albert Thomas rencontre à Paris Michel Fédoroff, qui préside le Comité central de patronage de la jeunesse russe à l'étranger et qui est en relation étroite avec le Zemgor. Fédoroff expose à cette occasion l'objet de son organisation : « reconstituer les forces intellectuelles russes qui ont été et continuent d'être implacablement détruites physiquement et moralement par le régime existant en Russie ». Selon lui, la formation des hommes qui seront appelés à diriger le rétablissement de l'économie nationale russe après l'épisode bolchevik incombe aux pays d'Europe occidentale et de l'Amérique du Nord. Cette formation doit se faire de deux manières : donner aux étudiants russes les moyens de poursuivre une formation universitaire avancée et leur trouver un emploi. Fédoroff demande donc au BIT d'assurer le placement des étudiants en fin d'étude « qui sont obligés de chercher du travail à l'étranger en attendant la possibilité de pouvoir rentrer dans leur patrie pour s'y consacrer à une activité pratique dans leur branche $»^{36}$. Jean Tcherkessov, le président du Comité central de l'Union des associations des étudiants russes émigrés développe la même argumentation : profiter des années d'exil à l'étranger pour s'instruire et recevoir la qualification pratique indispensable à un travail utile dans la Russie future. Il faut également maintenir « la culture nationale à l'étranger pour que les étudiants russes reviennent dans la patrie comme citoyens dignes de leur grand pays $»^{37}$.

La position du directeur du BIT à ce sujet est dictée par les limites du mandat de son organisation, ce qui implique, selon lui, que le problème des étudiants russes ne puisse être officiellement soulevé, cependant :

Bien que ce problème ne rentre pas directement dans la mission qui nous a été confiée, nous pouvons donner notre appui moral pour aider à secourir ou tirer d'embarras les étudiants russes. [...] Ils sont indispensables pour la reconstruction future de la Russie ${ }^{38}$.

\footnotetext{
34. En 1922, le gouvernement tchécoslovaque avait remis au secrétaire général de la SDN un mémoire distribué à tous les États membres, démontrant la connexion étroite entre la question des réfugiés et la question russe proprement dite. C'est à partir de cette date qu'il avait mis en place le système d'allocation afin de favoriser l'accès des jeunes réfugiés russes aux études supérieures.
}

35. N. Astrov au major Johnson, 10 janvier 1924. ASDN, FMN, R1738, 17822.

36. M. Fedoroff à A. Thomas, 3 mars 1925. ASDN, FMN, C1438, Rr 404/4/22/1.

37. J. Tcherkessov à A. Thomas, 21 mars 1925. ASDN, FMN, C1438, Rr 404/4/22/1 .

38. Note d'A. Thomas, 25 décembre 1924. ASDN, FMN, C1439, Rr 406/1/22/1. 
Ceci étant, quelle action concrète peut-elle être menée ? Le CCOP revient sur la question lors de sa session de septembre 1925 et obtient que le BIT conduise une enquête spécifique destinée à évaluer le nombre de personnes concernées. Notons que l'enquête concerne à la fois les étudiants réfugiés russes et arméniens, qui sont les deux populations bénéficiant du statut de réfugié à cette date. Les résultats sont présentés lors de la Conférence intergouvernementale sur la question des réfugiés de mai 1926 et discutés au sein du CCOP en septembre 1926. Les résultats de l'enquête ont été établis sur la base de recherches effectuées par les délégués du BIT et d'informations communiquées par les organisations communautaires de réfugiés et par l'International Student Service. Nous présentons ci-après uniquement les données concernant les étudiants russes ${ }^{39}$ :

\begin{tabular}{|c|c|c|c|c|c|}
\hline Pays & $\begin{array}{l}\text { Étudiants } \\
\text { réfugiés } \\
\text { russes } \\
\text { ayant } \\
\text { terminé } \\
\text { leurs études } \\
\text { en } 1925\end{array}$ & $\begin{array}{l}\text { Étudiants } \\
\text { réfugiés } \\
\text { russes } \\
\text { poursuivant } \\
\text { leurs études } \\
\text { en } 1925\end{array}$ & Pays & $\begin{array}{l}\text { Étudiants } \\
\text { réfugiés } \\
\text { russes } \\
\text { ayant } \\
\text { terminé } \\
\text { leurs études } \\
\text { en } 1925\end{array}$ & $\begin{array}{l}\text { Étudiants } \\
\text { réfugiés } \\
\text { russes } \\
\text { poursuivant } \\
\text { leurs études } \\
\text { en } 1925\end{array}$ \\
\hline Allemagne & 50 & 650 & Hongrie & - & 25 \\
\hline Autriche & 10 & 50 & Japon & - & 700 \\
\hline Belgique & 12 & 183 & Lettonie & - & 25 \\
\hline Bulgarie & - & 256 & Lituanie & - & 140 \\
\hline Chine & 32 & 380 & Norvège & - & 5 \\
\hline Chypre & 5 & 375 & Palestine & - & 5 \\
\hline Estonie & 715 & 1130 & Pologne & 175 & 108 \\
\hline Finlande & - & 559 & $\begin{array}{l}\text { Royaume des } \\
\text { Serbes, } \\
\text { Croates, } \\
\text { Slovènes }\end{array}$ & 6000 & 4200 \\
\hline France $^{40}$ & $\mathrm{NC}$ & $\mathrm{NC}$ & Suisse & $\mathrm{NC}$ & $\mathrm{NC}$ \\
\hline $\begin{array}{l}\text { Grande- } \\
\text { Bretagne }\end{array}$ & $\mathrm{NC}$ & $\mathrm{NC}$ & $\begin{array}{l}\text { Tchécoslova- } \\
\text { quie }\end{array}$ & 550 & 6500 \\
\hline Grèce & $\mathrm{NC}$ & $\mathrm{NC}$ & & & \\
\hline & & & TOTAL & 7549 & 15291 \\
\hline
\end{tabular}

Il serait vain de penser que ces informations puissent nous donner une image exacte du nombre et de la répartition des réfugiés russes en 1925. L'usage qui peut en être

39. Rapport sur la question des étudiants russes et arméniens soumis au Comité consultatif des organisations privées, 9 septembre 1926. ASDN, FMN, C 1470, A/C-2-1926.

40. Non communiqué. Le refus de la France de communiquer de manière officielle toute information chiffrée concernant les réfugiés russes et arméniens est une constante des documents genevois dans les années vingt. 
fait réside davantage dans la manière dont le problème des étudiants est présenté par les uns et perçu par les autres. Les organisations russes, le Zemgor en particulier, veulent démontrer que le problème des réfugiés est surtout à venir. Plus de quinze mille étudiants seront bientôt qualifiés, pour moitié spécialisés en lettres, droit ou théologie. Or, comme le constate le BIT, les pays d'accueil réservent les professions intellectuelles à leurs propres ressortissants et les professions libérales sont déjà encombrées. Comment assurer leur avenir professionnel? Si certains pays accueillent des immigrants dans les professions manuelles et techniques, ils craignent néanmoins, et avec raison, que les étudiants ne saisissent ensuite la première occasion pour quitter ces emplois et ne viennent « faire concurrence » aux nationaux dans une branche qui corresponde mieux à leurs capacités et aspirations. De fait, le BIT ne dispose que d'une marge d'action réduite en la matière. Albert Thomas va donc, au nom du BIT, demander à toutes les universités suisses ainsi qu'aux autorités fédérales des bourses pour les étudiants russes. Après plusieurs mois de négociation, il n'obtient qu'une dispense des droits d'inscription. Quant au placement professionnel, la pratique constatée va consister à généraliser autant que possible celle appliquée en Tchécosolovaquie : la mise en place pour les étudiants en fin d'étude d'une formation technique destinée à les rendre employables par les employeurs français, sous couvert de travailleurs manuels ou industriels. En brouillant la lisibilité des catégorisations du travail, les étudiants russes bénéficieront pour partie des opérations de placement du BIT jusqu'en 1929, mais il semble évident que les organisations de réfugiés sont sur cette question plus efficaces que le BIT.

\section{Considérations générales : structures non étatiques et espace transnational}

L'exemple du Zemgor et de ses rapports avec le Haut commissariat de la SDN pour les réfugiés ou le Service des réfugiés du BIT permet de dégager un certain nombre de remarques générales sur les relations entre organisation internationale et organisation non gouvernementale. Partons des points communs entre ces deux types de structure dans les années vingt, années qui furent celles de l'institutionnalisation du champ humanitaire international. Les deux entités opèrent de manière décentralisée, leurs limites sont mal définies spatialement ou en évolution constante, en fonction des concentrations de réfugiés pour l'une ou des opérations à mener pour l'autre. Les structures sont légères, voire dépourvues de réalité administrative stable. Le poids des hommes et la personnalisation de l'action sont caractéristiques. Considérons les relations entretenues entre le Zemgor et Albert Thomas. Ce dernier connaît Alexandre Konovalov (président du Zemgor à partir de 1925) depuis son séjour à Saint-Pétersbourg pendant la guerre. Il en est de même du prince Lvov qu'il connaît personnellement ${ }^{41}$. Par ailleurs, la première prise de contact pour une collaboration entre le BIT et le Zemgor concernant les opérations de placement

41. Prince Lvov à Albert Thomas, 30 septembre 1924. ASDN, FMN, C 1437, Rr 404/3/22/1. 
s'est effectuée dans les milieux des réformateurs sociaux européens. À Prague, lors du Congrès international de politique sociale, organisé à l'initiative de l'Association pour la protection légale des travailleurs et tenu du 30 septembre au 1er octobre 1924 devant près de 600 délégués, Albert Thomas, qui mène les discussions, plaide pour le dépassement de l'ère de la philanthropie et l'entrée dans celle de la « démocratie industrielle ${ }^{42}$. C'est à cette occasion qu'avec Vladimir Lebedeff il pose les jalons de la future collaboration entre les deux organisations ${ }^{43}$. Par la suite, cette personnalisation des relations s'incarne dans les contacts noués avec Michel Fédoroff. Le poids des individus et des contacts personnels est une réalité induite par l'empirisme qui caractérise l'action en faveur des réfugiés dans ces années-là, mais qui tient également à la propre conception d'Albert Thomas, homme de réseau s'il en est, pour qui le BIT doit être une « structure vivante » et non une administration désincarnée et anonyme. L'empirisme est également une caractéristique commune. Il y a là un enjeu de conjoncture car, pour l'organisation privée comme pour l'organisation internationale, il n'y a pas d'antériorité entre la doctrine et l'institutionnalisation. Les deux sont simultanées. Pour le BIT, c'est la volonté d'œuvrer pour une justice sociale internationale à partir de l'héritage de la pensée réformatrice de l'avant-guerre dont les logiques étaient d'abord nationales. Pour le Zemgor, c'est la nécessité de faire face à la réalité de l'État soviétique et d'un exil de longue durée. Il y a par contre capitalisation de pratiques antérieures pour le Zemgor comme pour le BIT : le premier se présente comme un organe fédérateur des différents comités locaux de l'avant-guerre, de la période révolutionnaire puis de la guerre civile ; le second est animé par un homme qui a développé pendant deux ans au ministère de l'Armement et des Équipements de guerre une pratique d'économie dirigée associant milieux patronaux et ouvriers. En ce qui concerne la codification juridique du statut de réfugié par le Haut commissariat de la SDN, on assiste à une situation comparable. La réflexion juridique sur les dénationalisations en masse ou sur le statut international de réfugié ne forment pas un corps doctrinaire préexistant aux réalités brutales de l'après-guerre, mais une conséquence de celles-ci. Le rôle central des juristes russes en atteste, comme d'ailleurs les débats et les polémiques qui agitent cette communauté face aux décisions genevoises ${ }^{44}$. L'empirisme est également d'ordre structurel car les deux organisations sont confrontées à une société internationale dominée par la seule légitimité des acteurs étatiques. Animés par une théorie de l'action, Albert Thomas comme Fridtjof Nansen se voient rappeler sans cesse lors des assemblées annuelles la précarité de leurs mandats ou de leurs budgets. Dès 1922, le BIT a été assigné par le gouvernement français devant la Cour permanente de justice internationale sur la question de

42. Les documents du travail, BIT, n91-92, nov.-déc. 1924, p. 233-250.

43. Note d'Albert Thomas, 8 octobre 1924. ASDN, FMN, C 1437, Rr 404/3/22/1.

44. La lecture des Poslednie novosti (Les Dernières nouvelles) de l'automne 1926 est à ce titre un bon exemple : la polémique tourne autour de l'imposition de la taxe de 5 francs-or sur les passeports Nansen des réfugiés russes et arméniens, taxe destinée à alimenter financièrement le placement professionnel des réfugiés par le BIT (articles, droits de réponse et lettres ouvertes de Nemanoff, Sidijkh, Rubinstein, Nolde, Goulkevitch et Zagorski). 
ses compétences en matière de travail agricole. Quant à l'organisation privée, sa dépendance est plus grande encore, les oscillations gouvernementales concernant les bureaux de placement du Zemgor en France le montrent clairement.

C'est ainsi qu'une organisation internationale spécialisée et une organisation communautaire de l'exil travaillent dans une même dimension déterritorialisée et transnationale. Pour le Zemgor, selon les termes employés par ses représentants à la SDN, la présence à l'étranger est vécue au départ comme une situation d'exil, donc anormale et devant cesser par la transformation de la situation politique du pays d'origine qui permettra le retour. Progressivement, le poids du temps qui passe, mais également les dynamiques propres qui animent les différentes organisations communautaires en l'absence de territoire modifient cette façon de penser. Ceci explique l'importance accordée à la question des étudiants. L'enjeu dépasse largement celui du travail ou du chômage dans les pays d'accueil, car c'est l'avenir politique de la Nation qui est engagé. Ainsi la trajectoire du Zemgor marque le passage d'administrations locales russes fédérées pendant la guerre à la création d'une organisation non gouvernementale dans l'exil par le réinvestissement de l'expérience, des pratiques et des contacts noués. Mais le Zemgor reste néanmoins une organisation atypique notamment dans les premières années, tant par sa capacité diplomatique et sa légitimité héritée, que par son personnel politique ou sa capacité financière. Cette atypie est-elle l'expression d'une modernité dans le domaine de l'action humanitaire ? Elle semble plutôt révélatrice d'une certaine faculté d'adaptation face aux nouvelles réalités et d'un rapprochement avec les expériences acquises par la diaspora juive européenne dans l'organisation d'une émigration en masse commencée dans les années 1880. L’importance du rôle joué par Lucien Wolf auprès du Haut commissaire Nansen, comme au sein du CCOP dans les années vingt en atteste. Il n'en reste pas moins que ces structures ont des intérêts propres qui ne se recoupent pas forcément. Les tentatives d'influences réciproques sont courantes au cours de la période de détermination des champs de légitimité.

Ainsi définies par leur situation marginale commune en terme d'acteurs de la société internationale, ces structures sont animées de logiques divergentes. L'organisation internationale se présente comme une interface reconnue et légitime dans ses rapports avec les États. C'est d'ailleurs pour cela que les organisations privées communautaires et généralistes appellent de leurs vœux la création d'une structure ad hoc par la SDN en 1921. C'est également dans cet objectif que le mandat du placement professionnel est confié au BIT lors de l'Assemblée générale de la SDN de septembre 1924. L'organisation privée fédératrice est, quant à elle, en interface avec une nébuleuse associative, dont elle tire sa légitimité et sa représentativité. Or, les champs politique et associatif des réfugiés russes sont le théâtre de clivages ou de rivalités internes en contradiction avec la volonté ou la nécessité vis-à-vis de l'interlocuteur étatique ou international de produire une image externe unitaire et un discours unique. Deux mythes de pouvoir et de reconnaissance se mêlent : celui de l'organisation internationale qui dispense un discours destiné à légitimer son aptitude à peser sur les décisions étatiques et celui de l'organisation privée tenue de faire de même par rapport à sa communauté de référence. Ainsi, Albert Thomas n'a 
pas les capacités opérationnelles nécessaires qui lui permettraient de mettre réellement en pratique les transferts et les placements des réfugiés. On observe à plusieurs reprises dans les archives qu'en réalité il ne contrôle pas les transferts faits en son nom par la Société générale d'immigration vers la France. Pour le Zemgor, c'est la confusion et les initiatives en tous sens en 1925 qui mettent en lumière les oppositions et les rivalités entre Prague et Paris, par exemple au moment même où chacun espère prendre le contrôle de l'opération de placement.

L'enjeu de légitimité et de reconnaissance n'est donc pas propre à l'organisation privée. Fridtjof Nansen a les mêmes difficultés et le Haut commissariat ne préexiste pas à ces organisations, c'est justement l'inverse. Il doit son existence comme les ressources de ces programmes d'action aux organisations privées, au contexte d'urgence humanitaire et au gain que certains États peuvent tirer de son existence (pour légitimer leur propre désistement, comme à Constantinople en 1921-1922). Il $\mathrm{y}$ a donc à la fois alliance stratégique et jeu de dupes dans les relations entre les deux types de structures. Les organisations privées usent de l'organisation internationale pour contourner l'opposition des États et mettre à profit sa capacité diplomatique et le caractère officiel de son action. Inversement, l'organisation internationale recourt aux organisations privées pour financer ses actions, justifier de leur caractère international et intervenir en tant qu'agents actifs, car elle n'a pas les moyens d'être directement opérationnelle.

$\mathrm{Au}$ lendemain du premier conflit mondial, la structuration du champ humanitaire est marquée par une complémentarité entre ces deux types d'organisation. Le Zemgor cumule dans ses rapports avec le Haut commissariat de la SDN et le Service des réfugiés du BIT trois types d'intervention : des pressions diversifiées, une vigilance normative et des actions opérationnelles. La coopération s'effectue de manière formelle au sein du Comité consultatif des organisations privées et de manière plus informelle et permanente par les contacts personnels noués avec Albert Thomas ou Fridtjof Nansen. Il faudra attendre la période après 1945 pour voir ces liens s'institutionnaliser. L'article 71 de la Charte des Nations-Unies prévoit que les organisations privées (ONG) puissent être consultées par le Conseil économique et social. Un statut « consultatif » peut être accordé par l'organisation intergouvernementale à une ou plusieurs ONG qui ont ainsi statutairement la possibilité de présenter leur position ou desiderata. Aujourd'hui au sein du Haut commissariat des Nations-Unies, les ONG sont, quantitativement, les partenaires les plus importants. Un quart du budget de l'organisation transite par elles pour mener à bien les actions opérationnelles. On ne peut donc que constater la montée en puissance de l'organisation internationale pour atteindre une situation exactement inverse à celle des années vingt.

\section{Université Paris-X Nanterre}

dzovinar.kevonian@wanadoo.fr 